\title{
VOC Sorption in Stretched Cross-Linked Natural
}

\section{Rubber}

Nikola Gushterov ${ }^{\dagger}$, Ferruccio Doghieri ${ }^{\ddagger}$, Dominik Quitmann ${ }^{\S}$, Elisabeth Niesing ${ }^{\dagger}$, Frank Katzenberg ${ }^{\S}$, Jorg C. Tiller ${ }^{\S}$ and Gabriele Sadowski ${ }^{*}$,

${ }^{\dagger}$ Laboratory of Thermodynamics, Department of Biochemical \& Chemical Engineering, TU Dortmund, Emil-Figge-Str. $\quad 70, \quad 44227 \quad$ Dortmund, Germany

* Dipartimento di Ingegneria Civile, Chimica, Ambientale e dei Materiali, Università di Bologna, $\begin{array}{lllll}\text { Via } & \text { Terracini } & 28, & 40131 & \text { Bologna, }\end{array}$

${ }^{\S}$ Chair of Biomaterials \& Polymer Science, Department of Biochemical \& Chemical Engineering, TU Dortmund, Emil-Figge-Str. 66, 44227 Dortmund, Germany

\section{Corresponding Author}

*E-mail: gabriele.sadowski@bci.tu-dortmund.de 
Table S1. Toluene-vapor sorption data at $293.15 \mathrm{~K}$ in NR, cross-linked with $0.2 \mathrm{phr}$ DCP stretched at different extension ratios $\lambda$.

\begin{tabular}{|c|c|c|c|}
\hline \multirow{2}{*}{$\begin{array}{c}\text { Pressure, } \\
p(\text { mbar })\end{array}$} & \multicolumn{3}{|c|}{ Toluene weight fraction $w$} \\
\cline { 2 - 4 } & $\begin{array}{c}\lambda=1 \\
\text { (non-stretched) }\end{array}$ & $\lambda=7$ & $\lambda=10$ \\
\hline 3 & 0.029 & 0.025 & 0.018 \\
\hline 6 & 0.060 & 0.052 & 0.039 \\
\hline 9 & 0.093 & 0.086 & 0.067 \\
\hline 12 & 0.133 & 0.125 & 0.095 \\
\hline 15 & 0.183 & 0.171 & 0.137 \\
\hline 18 & 0.242 & 0.229 & 0.190 \\
\hline 21 & 0.311 & 0.306 & 0.271 \\
\hline 24 & 0.421 & 0.420 & 0.376 \\
\hline
\end{tabular}


Table S2. Chloroform-vapor sorption data at $293.15 \mathrm{~K}$ in NR, cross-linked with $0.2 \mathrm{phr}$ DCP stretched at different extension ratios $\lambda$.

\begin{tabular}{|c|c|c|c|}
\hline \multirow{2}{*}{$\begin{array}{c}\text { Pressure, } \\
p(\text { mbar })\end{array}$} & \multicolumn{3}{|c|}{ Chloroform weight fraction $w$} \\
\cline { 2 - 4 } & $\begin{array}{c}\lambda=1 \\
\text { (non-stretched) }\end{array}$ & $\lambda=7$ & $\lambda=10$ \\
\hline 22 & 0.057 & 0.050 & 0.044 \\
\hline 44 & 0.116 & 0.105 & 0.091 \\
\hline 66 & 0.176 & 0.165 & 0.150 \\
\hline 88 & 0.240 & 0.229 & 0.218 \\
\hline 110 & 0.309 & 0.304 & 0.286 \\
\hline 132 & 0.385 & 0.389 & - \\
\hline 154 & 0.474 & - & - \\
\hline 176 & 0.583 & - & - \\
\hline
\end{tabular}


Table S3. Acetone-vapor sorption data at $293.15 \mathrm{~K}$ in NR, cross-linked with $0.2 \mathrm{phr}$ DCP stretched at different extension ratios $\lambda$.

\begin{tabular}{|c|c|c|}
\hline \multirow{2}{*}{$\begin{array}{c}\text { Pressure, } \\
p \text { (mbar) }\end{array}$} & \multicolumn{2}{|c|}{ Acetone weight fraction $w$} \\
\cline { 2 - 3 } & $\begin{array}{c}\lambda=1 \\
\text { (non-stretched) }\end{array}$ & $\lambda=10$ \\
\hline 25 & 0.005 & 0.004 \\
\hline 50 & 0.011 & 0.008 \\
\hline 75 & 0.018 & 0.013 \\
\hline 100 & 0.026 & 0.019 \\
\hline 125 & 0.036 & 0.027 \\
\hline 150 & 0.047 & 0.038 \\
\hline 175 & 0.063 & 0.050 \\
\hline 200 & 0.085 & 0.066 \\
\hline
\end{tabular}

